\title{
Salt and water: not so simple
}

\author{
Mark L. Zeidel \\ Nephrology Division and Department of Medicine, Beth Israel Deaconess Medical Center and Harvard Medical School, Boston, Massachusetts, USA
}

\begin{abstract}
It has long been viewed that the maintenance of osmotic balance in response to high salt intake is a passive process that is mediated largely by increased water consumption to balance the salt load. Two studies in this issue of the $\mathrm{JCl}$ challenge this notion and demonstrate that osmotic balance in response to high salt intake involves a complex regulatory process that is influenced by hormone fluctuation, metabolism, food consumption, water intake, and renal salt and water excretion. Rakova et al. report the unexpected observation that long-term high salt intake did not increase water consumption in humans but instead increased water retention. Moreover, salt and water balance was influenced by glucocorticoid and mineralocorticoid fluctuations. Kitada et al. extend upon these findings in mouse models and determined that increased urea and a corresponding increase in urea transporters in the renal medulla as the result of increased protein intake promote the water retention that is needed to achieve osmotic homeostasis. Together, the results of these two studies lay the groundwork for future studies to determine how, in the face of chronic changes in salt intake, humans maintain volume and osmotic homeostasis.
\end{abstract}

\section{Maintaining salt and water balance}

The great French biologist Claude Bernard equated the body fluids with the ancient oceans that we all evolved from and asserted that the "constancy of the internal milieu is the condition of a free and independent existence," that is, the ability to live on land, apart from the primal seas (1). Since the time of Bernard, physiologists and physicians have worked to understand how we maintain stable osmolality and overall circulatory volume in the face of massive shifts in salt intake. On the basis of classical balance studies in humans and experimental animals (2), generations of renal physiologists viewed osmotic homeostasis during salt loading as a passive, reactive process. In this view, salt loading stimulates thirst by increasing blood osmolality, which in turn is sensed in the subfornical organ (SFO) of the brainstem. Along with the salt load, increased water intake raises extracellular fluid volume, elevates cardiac preload and cardiac output, and increases renal plasma flow and the glomerular filtration rate, thereby driving sufficient renal salt excretion to rebalance extracellular volume. As the kidney excretes the salt load, antidiuretic hormone-driven (ADH-driven) distal nephron free water reabsorption maintains osmotic homeostasis.

Two companion articles in this issue upend this classical narrative and reveal a complex interplay between the regulated metabolism of salt, water, protein, and fat, all of which are required for the maintenance of salt balance and osmotic homeostasis in the face of a chronic salt load. In the first study, Rakova et al. (3) took advantage of the planned isolation of Russian cosmonauts for a simulated long-duration space flight to evaluate, over the long term, the impact of changes in salt intake on water and salt excre-

\section{Related Articles: pp. 1932 and 1944}

tion. The authors were startled to find that chronic high salt intake resulted in less, not more, fluid intake and that salt and water balance were influenced by rhythmic variations in glucocorticoid and mineralocorticoid release. Salt excretion was accompanied by free water retention, and while salt excretion rose, excretion of urea and potassium was reduced. Rakova and colleagues concluded that urea and potassium substituted for sodium in the medullary interstitium as the osmotic drivers of water reabsorption in the cosmonauts. The second study by Kitada et al. (4) replicated these findings in mice to explore the mechanisms behind the findings of Rakova and colleagues. Increased salt intake in mice similarly augmented salt excretion and failed to increase water intake. In the face of increased salt excretion, the free water retention needed for osmotic homeostasis was achieved by increased accumulation of urea in the renal medulla, which, in turn, arose from increased protein intake and catabolism, as well as through an upregulation of renal urea transporters.

The findings of these combined studies are important, because the careful measurements describe in detail the physiological events underlying chronic salt regulation in humans. Moreover, this work reveals the complex orchestration of adrenal hormone release, sympathetic nerve outflow, metabolism in muscle, fat, and liver, and renal handling of salt and water needed for excretion of a major salt load while maintaining stable blood osmolality.

In some respects, the lack of increase in water intake in response to the salt load is not surprising. Studies in normal humans have shown that acute increases in serum osmolality effected by infusion of hypertonic saline reliably result in rapid increases in $\mathrm{ADH}$ release, while the perception of thirst is variable and occurs at a higher serum osmolality (5). In this regard, it is interesting that urine osmolalities in the cosmonauts on high-salt diets did not rise to maximal levels, suggesting that serum osmolalities rarely rose to a level 
high enough to stimulate maximal $\mathrm{ADH}$ release and may not have risen enough to drive thirst (3). Sleep provides another example of the primacy of ADH release over thirst in response to small increases in serum osmolality. During sleep, insensible water loss starts to raise serum osmolality, which triggers $\mathrm{ADH}$ release, and increases renal reabsorption of free water, which corrects serum osmolality without the need for arousal and thirst to drive drinking. Similarly, in chronically salt-loaded mice and humans, the kidney's ability to concentrate urine, coupled with the generation of free water from increased protein and fat metabolism, was more than sufficient to maintain stable blood osmolality during salt loading $(3,4)$.

\section{Unanswered questions}

If osmolality must rise quite substantially before thirst is engaged, then how is thirst meshed with the fine control of serum osmolality? For that matter, where and how do organisms orchestrate the multiple control mechanisms (hormone release, metabolism, and excretion of renal salt and water) shown by the two articles in this issue? Can future studies define these control mechanisms with clarity? The brainstem, hypothalamus, and structures of the lamina terminalis (organum vasculosum laminae terminalis [OVLT], median preoptic nucleus [MnPO], and SFO) control salt balance and osmotic homeostasis and integrate external environmental stimuli to manage nearly all aspects of homeostasis. Because these regions have so many different neurons in such close proximity, it has been difficult to map out neural control circuits in them. Newer methods that combine genetic targeting with stereotactic localization of interventions and measurements permit monitoring and manipulation of specific neuronal types in specific locations, often in conscious, freely moving mice $(6,7)$. The very recent use of high-throughput single-cell transcriptomic technologies, such as Drop-seq, to define with great precision the different populations of neurons within anatomic regions of the brain will likely lead to the identification of ever more specific neuron populations, which can then be targeted genetically (8). Using these approaches, investigators have mapped with increasing definition the control within brain neurons of appetite and satiety (9), sleep and wakefulness (10), bladder function (11), as well as thirst and ADH release $(12,13)$.

Recent studies on the control of SFO neurons that regulate thirst revealed that the level of activation of these neurons is determined by serum osmolality, which they sense directly, and, at much higher sensitivity, by their estimate of the impact that drinking water or eating food will have on serum osmolality (12). Thus, water deprivation activates SFO neurons, and initiation of drinking reduces this activation, long before water ingestion alters serum osmolality. Additionally, food intake almost immediately activates SFO neurons, long before food ingestion alters serum osmolality. Similarly, ADHreleasing neurons in the hypothalamus are activated immediately by eating and are inhibited immediately by drinking and by the anticipation of drinking (13). Thus, we are beginning to understand how the brain integrates environmental cues with the homeostatic needs of the organism, so that in the future we can begin to explain, for example, why the cosmonauts did not drink more in response to salt loading, but instead accelerated catabolism and renal free water reabsorption.

The studies by Rakova et al. and Kitada et al. demonstrate that controlling salt excretion and osmotic homeostasis in the face of wide variations in salt load engages the release of hormones such as glucocorticoids and mineralocorticoids, alters filtration and tubular handling of sodium and water in the nephron, changes protein and fat metabolism, and alters drinking and eating. The neural circuits that adapt intake and excretion, as well as metabolism, to the ongoing needs of the organism in its environment are just being revealed. As these pathways become clearer, therapies targeted to the control of specific neural circuits may be possible and will permit improved treatment of conditions like hypertension, congestive heart failure, and hyponatremia.

\section{Acknowledgments}

The author's work is supported by the NIH (P20 DK 108276 and RO-1 DK 113030). The author thanks Martin Pollak, Brad Lowell, Ananth Karumanchi, Seth Alper, Warren Hill, Bryce MacIver, and John Mathai (all from Department of Medicine, Beth Israel Deaconess Medical Center and Harvard Medical School, Boston, MA, USA) for their helpful comments.

Address correspondence to: Mark L. Zeidel, Nephrology Division and Department of Medicine, Beth Israel Deaconess Medical Center and Harvard Medical School, 330 Brookline Avenue, Boston, Massachusetts 02215, USA. Phone: 617.667.5260; E-mail: mzeidel@bidmc.harvard.edu.

1. Bernard C. Leçons Sur Les Propriétés Physiologiques Et Les Altérations Pathologiques Des Liquides De L'organisme. Paris, France: J.B. Bailleère; 1859.

2. Hall JE, Guyton AC, Smith MJ, Coleman TG. Blood pressure and renal function during chronic changes in sodium intake: role of angiotensin. Am J Physiol. 1980;239(3):F271-F280.

3. Rakova N, et al. Increased salt consumption induces body water conservation and decreases fluid intake. J Clin Invest. 2017; 127(5):1932-1943.

4. Kitada K, et al. High salt intake reprioritizes osmolyte and energy metabolism for body fluid conservation. J Clin Invest. 2017;127(5):1944-1959.

5. Zerbe RL, Robertson GL. Osmoregulation of thirst and vasopressin secretion in human subjects: effect of various solutes. Am J Physiol. 1983;244(6):E607-E614.

6. Roth BL. DREADDs for neuroscientists. Neuron. 2016;89(4):683-694.

7. Rajasethupathy P, Ferenczi E, Deisseroth K. Targeting neural circuits. Cell. 2016;165(3):524-534.

8. Campbell JN, et al. A molecular census of arcuate hypothalamus and median eminence cell types. Nat Neurosci. 2017;20(3):484-496.

9. Fenselau $\mathrm{H}$, et al. A rapidly acting glutamatergic $\mathrm{ARC} \rightarrow \mathrm{PVH}$ satiety circuit post synaptically regulated by $\alpha$-MSH. Nat Neurosci. 2017;20(1):42-51.

10. Vetrivelan R, et al. Melanin-concentrating hormone neurons specifically promote rapid eye movement sleep in mice. Neuroscience. 2016;336:102-113.

11. Hou XH, et al. Central control circuit for context-dependent micturition. Cell. 2016;167(1):73-86.

12. Zimmerman CA, et al. Thirst neurons anticipate the homeostatic consequences of eating and drinking. Nature. 2016;537(7622):680-684.

13. Mandelblat-Cerf Y, et al. Bidirectional anticipation of future osmotic challenges by vasopressin neurons. Neuron. 2017;93(1):57-65. 